PAPER

\title{
Headache as the only neurological sign of cerebral venous thrombosis: a series of 17 cases
}

\author{
R Cumurciuc, I Crassard, M Sarov, D Valade, M G Bousser
}

See Editorial Commentary, p 1043

J Neurol Neurosurg Psychiatry 2005;76:1084-1087. doi: 10.1136/jnnp.2004.056275

See end of article for authors' affiliations

.....................

Correspondence to:

Dr R Cumurciuc,

Department of Neurology, Lariboisiere hospital, 2 rue Ambroise Pare, 75010

Paris, France; rodica. cumurciuc@|rb.ap-hopparis.fr

Received 22 October 2004 Revised version received 17 December 2004

Accepted

21 December 2004
Background: Headache is the most frequent symptom in cerebral venous thrombosis (CVT), and usually the first. However, it has rarely been reported as the only symptom of CVT.

Objectives: To study the characteristics of patients in whom headache was the only presentation of CVT in the absence of intracranial hypertension, subarachnoid haemorrhage (SAH), meningitis, or other intracranial lesion.

Methods: From a prospective study of 123 consecutive patients with CVT only those with isolated headache and normal brain computed tomography (CT) scan and cerebrospinal fluid (CSF) examination were included in the present study. All patients underwent an extensive systematic aetiological work-up and were given intravenous heparin followed by oral anticoagulants. A detailed description of the headache was obtained.

Results: Headache was only sign of CVT in 17 patients. The lateral sinus was the most frequently involved sinus $(n=15)$. Onset of headache was progressive in 11 , acute in 3 , and thunderclap in 3 patients. Once established, the headache was continuous in 15, diffuse in four and unilateral in 13, usually ipsilateral to the occluded lateral sinus. No specific risk factor or cause was found. All had a favourable evolution. Conclusion: The pathogenesis of isolated headache in CVT in the absence of intracranial hypertension, $\mathrm{SAH}$, meningitis or intracerebral lesion is unknown but may involve changes in the walls of the occluded sinus. Hence MRI/MRV should be used to look for signs of CVT in all patients with recent headache (progressive or thunderclap) even when the CT scan and CSF examination are normal.

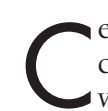
erebral venous thrombosis (CVT) is an uncommon cerebrovascular disease presenting with a remarkably wide spectrum of signs and mode of onset. ${ }^{1}$ In all series, headache is the most frequently occurring symptom at any time, present in over $80 \%$ of cases, ${ }^{1}$ and it is also the commonest initial symptom. The headache has no specific features and is almost invariably associated with other neurological signs such as papilloedema, focal deficits, seizures, disorders of consciousness, or cranial nerve palsies. These signs can be grouped into four main syndromes: isolated intracranial hypertension, focal syndrome, diffuse encephalopathy, and cavernous sinus syndrome. ${ }^{1-4}$ In rare cases of CVT, the headache is not accompanied by any clinical findings, but brain computed tomography (CT) scanning and/or cerebrospinal fluid (CSF) examination usually reveal conditions that explain the headache, such as subarachnoid haemorrhage (SAH), intracerebral infarction or haemorrhage due to the CVT, or meningitis related to the aetiology of CVT. Headache as the only presentation of CVT in the absence of such conditions is rare. ${ }^{5-8}$

In this paper we present the characteristics of 17 patientsfrom a prospective series of 123 patients with CVT seen over four years at our institution-in whom headache was the only clinical presentation of CVT in the absence of intracranial hypertension, SAH, meningitis, or other intracranial lesion as assessed by head CT and/or CSF examination.

\section{PATIENTS AND METHODS}

The present study is based on a prospective cohort of 123 consecutive patients with CVT admitted to our department between December 1999 and July 2004, of whom 28 were included in the International Study on Cerebral Vein and Dural Sinus Thrombosis (ISCVT). . Diagnosis of CVT was based on magnetic resonance imaging (MRI) combined with
MR venography (MRV), and/or helical CT venography and/or conventional angiography.

Patients were included in the present study if headache was the only manifestation of CVT in the absence of intracranial hypertension (no papilloedema and/or normal CSF pressure), SAH (no blood on CT scan and CSF examination), meningitis (normal CSF), or any intracerebral lesion on CT scan and/or MRI.

We obtained a detailed description of the headache including its mode of onset, location, severity, pattern, and evolution. The headaches were classified into different types according to the criteria of the International Headache Society (IHS). For all patients we recorded the past history of the headache and residual headache three months after CVT. A visual analogue scale (VAS) was used to record pain severity ( severe headache $=$ VAS $\geqslant 7$ ). Three modes of onset of the headache were defined depending on the time between the initial pain sensation and the most severe headache:

- "thunderclap"-sudden onset of an excruciating headache (VAS more than 8/10), reaching maximum intensity in less than one minute, and lasting more than one hour

- acute headache developing in less than 24 hours

- progressive over 24 hours. After 24 hours, the pain was characterised as continuous or intermittent (with headache free periods).

Abbreviations: CT, computed tomography; CSF, cerebrospinal fluid; CVT, cerebral venous thrombosis; ISCVT, International Study on Cerebral Vein and Dural Sinus Thrombosis; MRI/V, magnetic resonance imaging/venography; SAH, subarachnoid haemorrhage; VAS, visual analogue scale 
All patients underwent an extensive systematic aetiological work-up in which we looked for the main causes and risk factors of CVT such as systemic diseases, malignancies, haematologic disorders, antiphospholipid syndrome, and local (infectious or non-infectious) causes. Treatment was based on intravenous unfractionated heparin, followed by oral anticoagulation for six months or longer according to the aetiology of CVT. All the patients were prescribed analgesics or narcotics according to the severity of their headache.

\section{RESULTS}

Of $28 / 123$ patients $(23 \%)$ who presented with headache as only neurological symptom on admission, 17 (14\%; six men and 11 women; mean age 37.6 years, range $22-5$; table 1 ) satisfied our inclusion criteria. Eight patients (47\%) were recruited through the emergency headache centre and nine were admitted directly to the neurology unit.

The mean delay between onset and diagnosis was 13.1 days (range 0-30). The shortest delay (less than 24 hours) was observed in two patients with thunderclap headache and the longest in another two patients, of whom one had a progressive and the other an acute headache. One of the longest delays occurred in a patient with several episodes of thunderclap headache. Predisposing factors or causes of CVT were as follows: current oral contraceptive use $(\mathrm{n}=9)$, antiphospholipid antibody syndrome with systemic lupus erythematosus $(n=2)$, thrombophilia due to protein $S$ deficiency and/or factor V Leiden mutation $(n=3)$, and iron deficiency anaemia $(n=2)$. No cause was found in four patients, two of whom had a history of recurrent venous thrombosis. The sinus most frequent involved was the lateral sinus $(n=15)$, either isolated $(n=8)$ or in association with jugular vein thrombosis $(n=4)$ or with other sinuses $(n=3)$. The superior sagittal sinus was involved in four patients (isolated in two and associated with lateral sinus and deep venous system in the other two). An initial non-contrast CT scan was performed in 15 patients. It showed a definite spontaneous hyperdensity of one or several sinuses in nine patients and was normal in six patients (fig 1).

The main headache characteristics are given in table 2 . Four patients had a history of migraine without aura before CVT. In three the headache differed from their usual

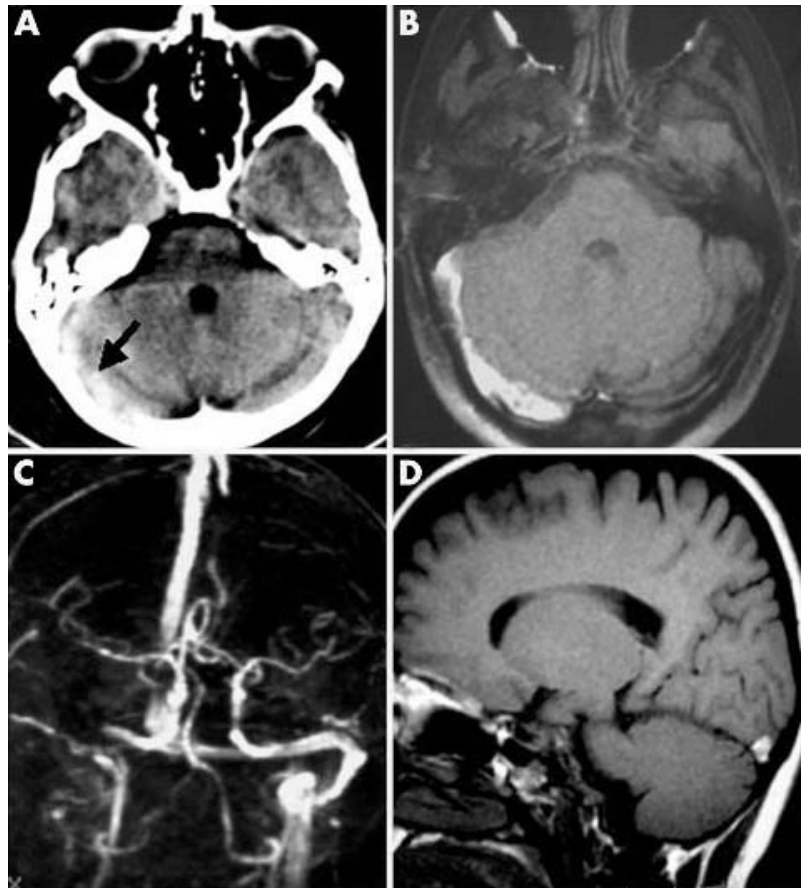

Figure 1 Patient 6. (A) Non-contrast cerebral computed tomography scan showing spontaneous hyperdensity of the right lateral sinus (arrow). (B-D) Cerebral MRI/MRV: hypersignal of the right lateral sinus on (B) axial fluid attenuated inversion recovery (FLAIR) images and (D) on sagittal T1-weighted images. (C) Right lateral sinus and jugular vein were not seen on cerebral MRV, confirming thrombosis.

migraine, and the time from onset to diagnosis was six to seven days. One patient had a headache that was initially similar to her usual migraine attacks but it persisted. She was evaluated 23 days after onset. The onset of headache was progressive over a few days in $65 \%(n=11)$, acute in $17.5 \%$ $(\mathrm{n}=3)$, and thunderclap in $17.5 \%(\mathrm{n}=3)$ of the patients. Once established, the headache was continuous in $88 \%(n=15)$; two of these had superimposed attacks of

Table 1 Baseline characteristics of patients with isolated headache as only sign of CVT

\begin{tabular}{|c|c|c|c|c|c|c|}
\hline Patient & Sex & Age & $\begin{array}{l}\text { Time from onset to } \\
\text { diagnosis (days) }\end{array}$ & Predisposing factor/cause & Site of thrombosis & Non-contrast CT scan \\
\hline 1 & $M$ & 40 & $<1$ & No & SSS, RLS, DVS & Spontaneous hyperdensity \\
\hline 2 & $\mathrm{~F}$ & 26 & 23 & Oral contraception & SSS, RLS, DVS & Spontaneous hyperdensity \\
\hline 3 & M & 40 & 2 & Recurrent venous thrombosis & LLS & - \\
\hline 4 & $\mathrm{~F}$ & 41 & 15 & $\mathrm{SLE}+\mathrm{APL}$ & LLS, UV & Normal \\
\hline 5 & $\mathrm{~F}$ & 32 & $<1$ & Oral contraception & RLS, RJV & Spontaneous hyperdensity \\
\hline 6 & $\mathrm{~F}$ & 33 & 6 & Oral contraception & RLS, RJV & Spontaneous hyperdensity \\
\hline 7 & $\mathrm{~F}$ & 31 & 6 & Oral contraception & LLS & Spontaneous hyperdensity \\
\hline 8 & $\mathrm{~F}$ & 29 & 15 & $\begin{array}{l}\text { Gastric carcinoma, oral contraception, } \\
\text { iron deficiency anaemia }\end{array}$ & RLS & Normal \\
\hline 9 & $\mathrm{~F}$ & 26 & 30 & Oral contraception & RLS & Spontaneous hyperdensity \\
\hline 10 & $\mathrm{~F}$ & 30 & 20 & $\mathrm{SLE}+\mathrm{APL}$ & LLS, DVS & Spontaneous hyperdensity \\
\hline 11 & $M$ & 56 & 7 & Recurrent venous thrombosis & LLS & Spontaneous hyperdensity \\
\hline 12 & $\mathrm{~F}$ & 51 & 15 & $\begin{array}{l}\text { Oral contraception, factor V Leiden } \\
\text { mutation }\end{array}$ & LLS, LV & Normal \\
\hline 13 & M & 57 & 11 & $\begin{array}{l}\text { Protein-S deficiency, factor } V \text { Leiden } \\
\text { mutation }\end{array}$ & RLS & - \\
\hline 14 & $\mathrm{~F}$ & 38 & 30 & Oral contraception & LLS & Normal \\
\hline 15 & M & 51 & 6 & Factor $V$ Leiden mutation & SSS & Normal \\
\hline 16 & $\mathrm{~F}$ & 22 & 27 & $\begin{array}{l}\text { Oral contraception, iron deficiency } \\
\text { anaemia }\end{array}$ & SSS & Normal \\
\hline 17 & M & 37 & 10 & No & RLS & Spontaneous hyperdensity \\
\hline
\end{tabular}

APL, antiphospholipid syndrome; DVS, deep venous system; F, female; UV, left jugular vein; LLS, left lateral sinus; M, male; RJV, right jugular vein; RLS, right lateral sinus; RVT, recurrent venous thrombosis; SLE, systemic lupus erythematosus; SSS, superior sagittal sinus. 
Table 2 Characteristics of the headache in patients with cerebral venous thrombosis

\begin{tabular}{|c|c|c|c|c|c|c|c|c|}
\hline Patient & History of migraine & Onset & Evolution & Pattern & $\begin{array}{l}\text { Severity } \\
\text { (VAS) }\end{array}$ & Location & Associated signs & Headache at 3 months \\
\hline 1 & No & $\mathrm{TH}$ & Continuous & Constrictive & 10 & Diffuse & Nausea and vomiting & No \\
\hline 2 & Migraine without aura & Progressive & Intermittent & Throbbing & 8 & $\mathrm{RHC}$ & No & Migraine without aura \\
\hline 3 & No & Acute & Continuous & Throbbing & 8 & LHC & Nausea and vomiting & No \\
\hline 4 & No & Progressive & Cluster-like attacks & Throbbing & 8 & LFO & Ocular redness & TTH \\
\hline 5 & No & $\mathrm{TH}$ & Continuous & Throbbing & 9 & $\mathrm{RHC}+\mathrm{RNP}$ & $\begin{array}{l}\text { Nausea and vomiting, phono/ } \\
\text { photophobia }\end{array}$ & Migraine with aura \\
\hline 6 & Migraine without aura & Progressive & Continuous & Throbbing & 9 & $\mathrm{RHC}+\mathrm{RNP}$ & No & Migraine without aura \\
\hline 7 & Migraine without aura & Progressive & Continuous & Throbbing & 9 & LHC & Nausea, phono/photophobia & $\mathrm{TH}+$ + migraine without aura \\
\hline 8 & No & Progressive & Continuous & Throbbing & 5 & RHC & Nausea, phono/photophobia & No \\
\hline 9 & No & Progressive & Continuous & Throbbing & 6 & RHC & Nausea and vomiting & No \\
\hline 10 & No & Progressive & Continuous & Throbbing & 10 & LFO & No & No \\
\hline 11 & No & Progressive & Continuous & Throbbing & 10 & LHC & Nausea and vomiting & No \\
\hline 12 & No & Progressive & Continuous & Throbbing & 9 & $L H C+L N P$ & No & $\pi \mathrm{H}$ \\
\hline 13 & No & Acute & Continuous & Constrictive & 9 & Diffuse & Nausea & No \\
\hline 14 & No & Acute & Continuous & Constrictive & 6 & LHC & Nausea and vomiting & Migraine without aura \\
\hline 15 & Migraine without aura & Progressive & Continuous + several episodes of TH & Throbbing & 9 & Diffuse & Nausea, phono/photophobia & $\begin{array}{l}\text { Migraine without aura + } \\
\text { episodic TH }\end{array}$ \\
\hline 16 & No & $\mathrm{TH}$ & Continuous + several episodes of $\mathrm{TH}$ & Throbbing & 9 & Diffuse & No & Migraine with aura \\
\hline 17 & No & Progressive & Continuous & Constrictive & 5 & $\mathrm{RHC}$ & No & No \\
\hline
\end{tabular}

thunderclap headache. Two patients had intermittent headache: one with unilateral and throbbing "migraine-like" headache, the other with "cluster-like" attacks of severe orbital pain lasting 30 minutes.

The headache was throbbing in $76 \%(\mathrm{n}=13)$ and severe in $76 \%(n=13)$ of the patients. It was diffuse in 4 , unilateral in 13, and associated with homolateral neck pain in 3 patients. Nausea, vomiting, and/or phono/photophobia was present in $59 \%(n=10)$. All patients except one with isolated unilateral lateral sinus thrombosis had a unilateral headache, ipsilateral to the thrombosis. One patient with right lateral sinus thrombosis had a diffuse headache. Patients with jugular vein thrombosis had ipsilateral neck pain.

In all patients, the severe headache started to improve in a few days. It disappeared within two weeks in two thirds of the patients and within one month in the rest. At three months, the four patients who had migraine before continued to have attacks of migraine and two had associated tension-type headache. A new onset of migraine with aura was observed in two patients and of tension-type headache in one.

\section{DISCUSSION}

In a prospective series of 123 patients seen over a four year period at our institution, $17(14 \%)$ had headache as the only manifestation of CVT in the absence of intracranial hypertension, SAH, meningitis, or intracranial lesions. This reflects a recruitment bias in favour of, firstly, CVT (our centre had the largest number of patients in ISCVT $^{9}$ ) and, secondly, headache (our emergency headache centre deals with 7500 patients per year).

A remarkable feature of this series is the unusually high frequency $(88 \%)$ of lateral sinus involvement either alone or associated with jugular vein or other sinus thrombosis. It is unlikely that isolated lateral sinus thrombosis was overdiagnosed because absence of flow on MRV was required in association with increased signal on MRI T1- and T2weighted images for the diagnosis. This allowed us to differentiate it from lateral sinus hypoplasia. ${ }^{10}$ Whether the isolated lateral sinus thrombosis would have continued to remain isolated or whether it was the initial manifestation of a thrombotic process that might have extended to other sinuses remains debatable. However, it is noteworthy that the delay between the onset and diagnosis was not significantly different in the patients with isolated lateral sinus thrombosis from the patients with lateral sinus thrombosis associated with other sinuses. In contrast, superior sagittal sinus thrombosis (the superior sagittal sinus is the most frequently involved in large CVT series) was detected in only four patients, most likely because headache in superior sagittal sinus thrombosis is associated with signs of intracranial hypertension, focal deficits, or disorders of consciousness. This also equally applies to bilateral lateral sinus thrombosis which usually induces intracranial hypertension. Apart from the site of thrombosis, all other characteristics (age, sex, aetiology) were similar to those of CVT in general. By definition, the prognosis was good, but again, it is not possible to conclude if the patients would have recovered spontaneously or whether heparin played a favourable role.

There was no uniform pattern of headache in this series. The most frequent characteristics were: recent rapidly progressive onset (less than 30 days) of severe headache (VSA above 8/10); persistent course (15/17); unilateral location (13/17); and throbbing quality (13/17). Three patients presented with thunderclap headache: one had superior sagittal sinus thrombosis, one had extensive thrombosis (superior sagittal sinus, lateral sinus, and deep venous system) and one had lateral sinus and jugular vein thrombosis. So thunderclap headache may occur in CVT in the absence of subarachnoid or intracerebral haemorrhage. Two of these patients had a spontaneous hyperdense sinus on CT scan that was suggestive of CVT, but the third had a completely normal CT scan. This demonstrates the importance of MRI/MRV in patients who have a thunderclap headache with normal CT scan and CSF examination. In two patients the headache was deceptive, mimicking migraine in one and cluster headache in the other.

Non-contrast cerebral CT scan is usually performed as the first investigation in patients with a recent persisting headache. The essential CT finding in our series was the spontaneous hyperdensity of the thrombosed sinus, present in $60 \%$ of patients. This sign, known as the "dense triangle" in superior sagittal sinus thrombosis, ${ }^{11}$ may be seen in any sinus. However it is frequently missing or difficult to recognise with certainty, and there are false positives, particularly in children or in patients with haemoconcentration. ${ }^{12}{ }^{13}$ Hence, MRI/MRV is required when this sign is lacking or doubtful.

The mechanism of headache in CVT in the absence of intracranial hypertension, SAH, meningitis, or intracranial lesion is unknown. Stretching or irritation of nerve fibres in the walls of the occluded sinus is a possibility. It is also 
possible that a local inflammatory reaction occurs with dilatation of vessels in the sinus walls as suggested by the frequent contrast enhancement surrounding the clot, known as the "empty delta sign". ${ }^{14}$

In conclusion, isolated headache can be the only clinical sign of CVT in the absence of intracranial hypertension, SAH, meningitis or intracerebral lesion. In such cases CVT mostly involves a lateral sinus, either alone or in association with other sinuses. The headache is usually progressive over a few days, severe, persistent, unilateral and throbbing, but a few patients have sudden onset or even a thunderclap headache. The pathogenesis of the headache is unknown but may involve changes in the walls of the thrombosed sinus. Plain CT scan frequently shows a hyperdense sinus, but it is normal in $40 \%$ of patients. This points to a need for MRI/MRV in all patients with recent headache-progressive or thunderclapwith normal CT scan and CSF examination.

\author{
Authors' affiliations \\ R Cumurciuc, I Crassard, M G Bousser, Department of Neurology, \\ Lariboisière Hospital, Paris, France \\ M Sarov, D Valade, Department of Emergency Headache Centre, \\ Lariboisière Hospital, Paris, France \\ Competing interests: none declared
}

\section{REFERENCES}

1 Bousser MG, Ross Russell R. Cerebral venous thrombosis. Vol 1. London: WB Saunders, 1997.

2 Bousser MG, Good J, Kittner ST, et al. Headache associated with vascular disorders In: Silberstein SD, Lipton AB, Dalessio DJ, eds. Wolf's headache and other head pain. Oxford: Oxford University Press, 2001.

3 Biousse V, Ameri A, Bousser MG. Isolated intracranial hypertension as the only sign of cerebral venous thrombosis. Neurology 1999;53:1537-42.

4 Ameri A, Bousser MG. Cerebral venous thrombosis. Neurol Clin 1992;10:87-111

5 Saneto RP, Samples S, Kinkel RP. Traumatic intracerebral venous thrombosis associated with an abnormal golf swing. Headache 2000;40:595-8.

6 Stam BJ, Kappelle $\amalg$. Thunderclap headache as first symptom of cerebral venous thrombosis. Lancet 1996;348:1623-5.

7 Wasson J, Redenbaugh J. Transverse sinus thrombosis: an unusual cause of headache. Headache 1997;37:457-9.

8 Finelli PF, Carley MD. Cerebral venous thrombosis associated with epoetin alfa therapy. Arch Neurol 2000;57:260-2.

9 Ferro JM, Canhao P, Stam J, et al. Prognosis of cerebral vein and dural sinus thrombosis (ISCVT). Stroke 2004;35:664-70.

10 Mas JL, Meder JF, Meary E, et al. Magnetic resonance imaging in lateral sinus hypoplasia and thrombosis. Stroke 1990;21:1350-6.

11 Ford K, Sarwar M. Computed tomography of dural sinus thrombosis. AJNR Am J Neuroradiol 1981;2:539-43.

12 Segall HD, Ahmadi J, McComb JG, et al. Computed tomographic observations pertinent to intracranial venous thrombotic and occlusive disease in childhood. State of the art, some new data, and hypothesis. Radiology 1982;143:441-9.

13 Ben Salem D, Osseby GV, Rezaizadeh-Bourdariat K, et al. Spontaneous hyperdense intracranial vessels seen on CT scan in polycythemia cases. J Radiol 2003;84:605-8.

14 Shintani S, Murase H, Ota H, et al. "Pseudo-delta sign" on computed tomography in an extremely acute stage of superior sagittal sinus thrombosis - a case report. Angiology 1990;41:417-20. 